\title{
Exploring Low-Mass Dark Matter with CRESST
}

\author{
R. Strauss ${ }^{1}$ - G. Angloher ${ }^{1}$ - A. Bento ${ }^{2}$ - C. Bucci ${ }^{3}$ - L. Canonica ${ }^{3}$. \\ X. Defay ${ }^{4}$ - A. Erb $^{4,5}$ - F. v. Feilitzsch ${ }^{4}$ - N. Ferreiro Iachellini ${ }^{1}$ - P. Gorla ${ }^{3}$. \\ A. Güitlein ${ }^{6,7}$ - D. Hauff ${ }^{1}$ - J. Jochum ${ }^{8}$ - M. Kiefer ${ }^{1}$ - H. Kluck ${ }^{6,7}$. \\ H. Kraus ${ }^{9}$ - J. C. Lanfranchi ${ }^{4}$ - J. Loebell ${ }^{8}$ - A. Münster ${ }^{4}$ - C. Pagliarone ${ }^{3}$. \\ F. Petricca ${ }^{1}$ - W. Potzel $^{4}$ - F. Pröbst ${ }^{1}$ - F. Reindl ${ }^{1}$ - K. Schäffner ${ }^{3}$. \\ J. Schieck ${ }^{6,7}$ - S. Schönert ${ }^{4}$ - W. Seidel ${ }^{1}$ - L. Stodolsky ${ }^{1}$ - C. Strandhagen ${ }^{8}$. \\ A. Tanzke ${ }^{1}$ - H. H. Trinh Thi ${ }^{4}$ - C. Türkoglu ${ }^{6,7}$. M. Uffinger ${ }^{8}$ - A. Ulrich ${ }^{4}$. \\ I. Usherov $^{8}$ - S. Wawoczny ${ }^{4}$ - M. Willers ${ }^{4}$ - M. Wüstrich ${ }^{1}$ - A. Zöller ${ }^{4}$
}

Received: 30 September 2015 / Accepted: 7 January 2016 / Published online: 28 January 2016 (c) The Author(s) 2016. This article is published with open access at Springerlink.com

\begin{abstract}
The CRESST-II (Cryogenic Rare Event Search with Superconducting Thermometers) experiment, whose second phase has been successfully finished in summer 2015 , aims at the direct detection of dark matter particles. The intrinsic radiopurity of $\mathrm{CaWO}_{4}$ crystals, the capability to reject recoil events from alpha-surface contamination, and the energy threshold were significantly improved compared to previous runs of the experiment. A moderate exposure of $29 \mathrm{~kg}$-days acquired by one $\sim 250 \mathrm{~g}$ $\mathrm{CaWO}_{4}$ detector provides competitive limits on the spin-independent dark matter particle-nucleon cross section and probes a new region of parameter space for dark matter particle masses below $3 \mathrm{GeV} / \mathrm{c}^{2}$. The potential for low-mass dark matter particle search can be further exploited by a new detector design planned for CRESST-III. We describe the experimental strategy for the near future and give projections for the sensitivity.
\end{abstract}

\footnotetext{
R. Strauss

strauss@mpp.mpg.de

1 Max-Planck-Institut für Physik, 80805 Munich, Germany

2 Departamento de Fisica, Universidade de Coimbra, P3004 516 Coimbra, Portugal

3 INFN, Laboratori Nazionali del Gran Sasso, 67010 Assergi, Italy

4 Physik-Department and Excellence Cluster Universe, Technische Universität München, 85747 Garching, Germany

5 Walther-Meißner-Institut für Tieftemperaturforschung, 85748 Garching, Germany

6 Institut für Hochenergiephysik der Österreichischen Akademie der Wissenschaften, 1050 Wien, Austria

7 Atominstitut, Vienna University of Technology, 1020 Wien, Austria

8 Eberhard-Karls-Universität Tübingen, 72076 Tübingen, Germany

9 Department of Physics, University of Oxford, Oxford OX1 3RH, UK
} 
Keywords Dark matter - Cryogenic detector - Low-mass dark matter particles

\section{Introduction}

During the last two decades, direct searches for dark matter in the form of particles have rapidly improved their sensitivity to spin-independent dark matter particlenucleon scattering. Possible hints for low-mass dark matter particles with masses of $\mathcal{O}\left(10 \mathrm{GeV} / \mathrm{c}^{2}\right)$ which were published in recent years [1-4] are disfavoured or excluded by a variety of experiments [5-10]. These non-conclusive experimental results and new theoretical dark matter models have drawn the attention to dark matter particles with masses of $\sim 10 \mathrm{GeV} / \mathrm{c}^{2}$ and below [11].

\section{First Results from CRESST-II Phase 2}

The CRESST-II (Cryogenic Rare Event Search with Superconducting Thermometers) experiment uses scintillating $\mathrm{CaWO}_{4}$ crystals as target material for interactions with dark matter particles [1]. CRESST uses modular detectors of $\sim 300 \mathrm{~g}$ each of which is based on a two-channel detector readout: the $\mathrm{CaWO}_{4}$ target crystal is operated as a cryogenic detector at $\mathrm{mK}$ temperatures to measure the total deposited energy of particle interactions (phonon detector). The temperature rise is measured by a transition edge sensor (TES) which is made of a thin W film. A separate light detector, a siliconon-sapphire absorber (diameter $40 \mathrm{~mm}$, thickness: $500 \mu \mathrm{m}$ ), which is also equipped with a TES measures the scintillation light output induced by interactions in the target crystal. Due to light quenching [12] in $\mathrm{CaWO}_{4}$, the scintillation light detection allows to identify the type of particle interaction. This is crucial to discriminate unavoidable beta/gamma backgrounds from possible dark matter particle-induced nuclear-recoil events which, to a certain extent, can be even tagged as $\mathrm{O}, \mathrm{Ca}$, and $\mathrm{W}$ recoils [13].

CRESST-II Phase 2 was running between July 2013 and August 2015 with a total target mass of $\sim 5 \mathrm{~kg}$. Eighteen detector modules of four different detector designs [15] were operated. In this paper, the detector module (called TUM40) with the best overall performance is discussed. The target crystal, a block-shaped $\mathrm{CaWO}_{4}$ crystal with a mass of $248 \mathrm{~g}$, is held by sticks made of $\mathrm{CaWO}_{4}$ material [14]. Together with a polymeric foil (which covers the remaining part of the inner housing), the sticks provide a detector housing with a fully scintillating inner surface (see Fig. 1). With this detector, previously observed backgrounds from surface-alpha decays are rejected with high efficiency [14]. The $\mathrm{CaWO}_{4}$ crystal used (TUM40) was produced in-house at the Technische Universität München [16]. In this way, the intrinsic background level could be reduced to an average beta/gamma rate of $3.51 /(\mathrm{kg} \mathrm{keV}$ day $)$ at $1-40 \mathrm{keV}$ [17], a factor of 2-10 better than for previously available crystals. An energy threshold of $\sim 600 \mathrm{eV}$ and a resolution of $\sigma_{\mathrm{ph}}=(0.090 \pm 0.010) \mathrm{keV}$ (at $2.60 \mathrm{keV}$ ) were achieved with this detector.

In the first data of TUM40, all events observed in the region of interest for dark matter search are compatible with leakage of beta/gamma backgrounds. For the achieved exposure of $29 \mathrm{~kg}$-days, no indications for additional backgrounds or an excess signal are observed. Consequently, a limit on the spin-independent dark matter particle- 


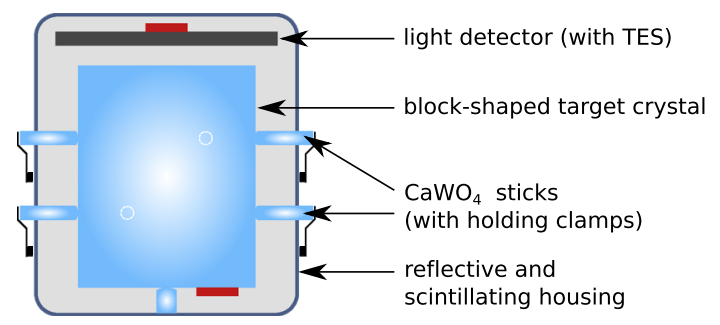

Fig. 1 Scheme of the new detector module: $\mathrm{CaWO}_{4}$ sticks are used to hold the target crystal. They are fed through holes in the copper support structure. Together with the scintillating foil surrounding the crystal, a fully scintillating inner detector housing is realized. This provides an efficient veto against surface-alpha backgrounds [14] (Color figure online)

nucleon scattering cross section is derived (see Fig. 3) [18]. It probes a new region of parameter space for dark matter particle masses below $3 \mathrm{GeV} / \mathrm{c}^{2}$ and disfavours the dark matter interpretation of the CRESST-II Phase 1 results [1].

\section{Potential of CRESST-III}

CRESST-II phonon detectors with masses of $\sim 300 \mathrm{~g}$ achieve energy thresholds of typically $300-800 \mathrm{eV}$ (50 \% trigger efficiency). Since the expected exponential recoil spectra extend only up to energies of $\mathcal{O}(1 \mathrm{keV})$ for dark matter particle masses of $\mathcal{O}\left(1 \mathrm{GeV} / \mathrm{c}^{2}\right)$ [11], the energy threshold is crucial for the sensitivity to low-mass dark matter.

Therefore, an enormous gain in sensitivity is expected if the energy resolution and, hence, the threshold of the phonon channel are reduced further. For CRESST-III, we chose a straight-forward approach to improve the detector performance, namely to scale down the volume of the absorber crystals. According to the model of cryogenic detectors [19], this has some basic implications for a calorimeter: For a crystal with reduced size, the area of TES can be smaller in order to absorb the same fraction of phonons induced by a certain energy $\Delta E$. Since the temperature rise in the TES $\Delta T \sim \Delta E / C$ increases when reducing the heat capacity $C$ of the thermometer, a smaller TES will consequently raise the signal-to-noise ratio of the detector. In addition, the collection area of the TES can be increased using superconducting Al phonon collectors (without increasing the heat capacity $C$ of the thermometer). Concretely, for a block-shaped $\mathrm{CaWO}_{4}$ with a size of $10 \times 20 \times 20 \mathrm{~mm}^{3}$ and a mass of $24 \mathrm{~g}$, an improvement in energy resolution by a factor of $10-50$ compared to $\sim 300 \mathrm{~g}$ crystals is expected. Therefore, energy thresholds of order $\mathcal{O}(50 \mathrm{eV})$ are feasible.

Parallel to us, an alternative concept to improve CRESST detectors was worked out [20]. Although completely unrelated to the CRESST-III strategy, we appreciate the findings in the manuscript, as they might become relevant for large-scale cryogenic experiments in the future.

According to the target crystal, the size of the light detector can be scaled down without a penalty in collection efficiency for scintillation photons. Since, in first approximation, the signal-to-noise ratio is inversely proportional to the volume of 
Fig. 2 Drawing of the CRESST-III detector prototype.

A $24 \mathrm{~g} \mathrm{CaWO}_{4}$ target crystal and a $20 \times 20 \mathrm{~mm}^{2}$ Si light detector are held by $\mathrm{CaWO}_{4}$ sticks

(Color figure online)

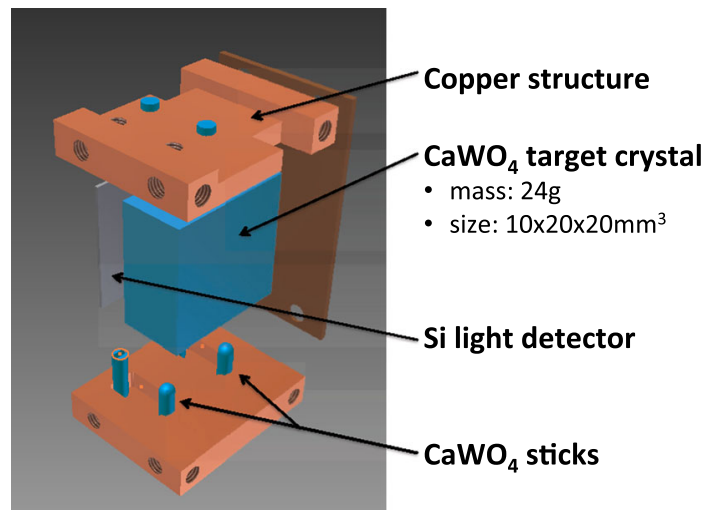

the absorber, an improvement by a factor of about 3-4 is expected for the CRESSTIII light detectors $\left(20 \times 20 \mathrm{~mm}^{2}\right)$ compared to that of CRESST-II (40 mm diameter). Furthermore, the re-absorption of photons in the $\mathrm{CaWO}_{4}$ crystal is reduced due to the smaller crystal size. At low energies $(\leq 10 \mathrm{keV})$, the light detector resolution is typically not sufficient to fully discriminate beta/gamma backgrounds. Since the light channel provides a partial discrimination of the background over the entire energy range down to the threshold, CRESST-III will still benefit moderately from improvements of the light channel.

In Fig. 2, a drawing of the CRESST-III detector prototype is shown. The target crystal and the light detector are held by $\mathrm{CaWO}_{4}$ sticks to establish a fully scintillating inner housing. This technique efficiently rejects all kinds of surface-alpha backgrounds [14].

An additional instrumentation of the detector holder, namely $\mathrm{CaWO}_{4}$ sticks equipped with TESs, is under investigation and will be the subject of future publications. The additional readout of events occurring in the sticks would reject all possible kinds of backgrounds related to the detector holder.

In summary, the following improvements in detector performance are assumed conservatively for CRESST-III:

- A phonon-detector energy threshold of $100 \mathrm{eV}$;

- Due to the reduced size of light detector (by a factor of $\sim 3$ ), an improvement in resolution by a factor of 3 ;

- Due to the reduced thickness of the crystal (reduced absorption of photons in the crystal), an increase in light collection by a factor of 2 .

The response of such a detector is computed by MC simulations [18] based on the same background level as in CRESST-II Phase 2. The resulting limit on the spinindependent dark matter particle-nucleon cross section is expected between the dashed $1 \sigma$ lines in Fig. 3 (yellow band) for an exposure of $50 \mathrm{~kg}$-days. The presently best limits would be lowered by about three orders of magnitude below $\leq 5 \mathrm{GeV} / \mathrm{c}^{2}$. The desired exposure could be achieved by operating ten CRESST-III detectors of $24 \mathrm{~g}$ each for $\sim 1$ year. A higher exposure does not give a significant improvement unless lowering the background level. 


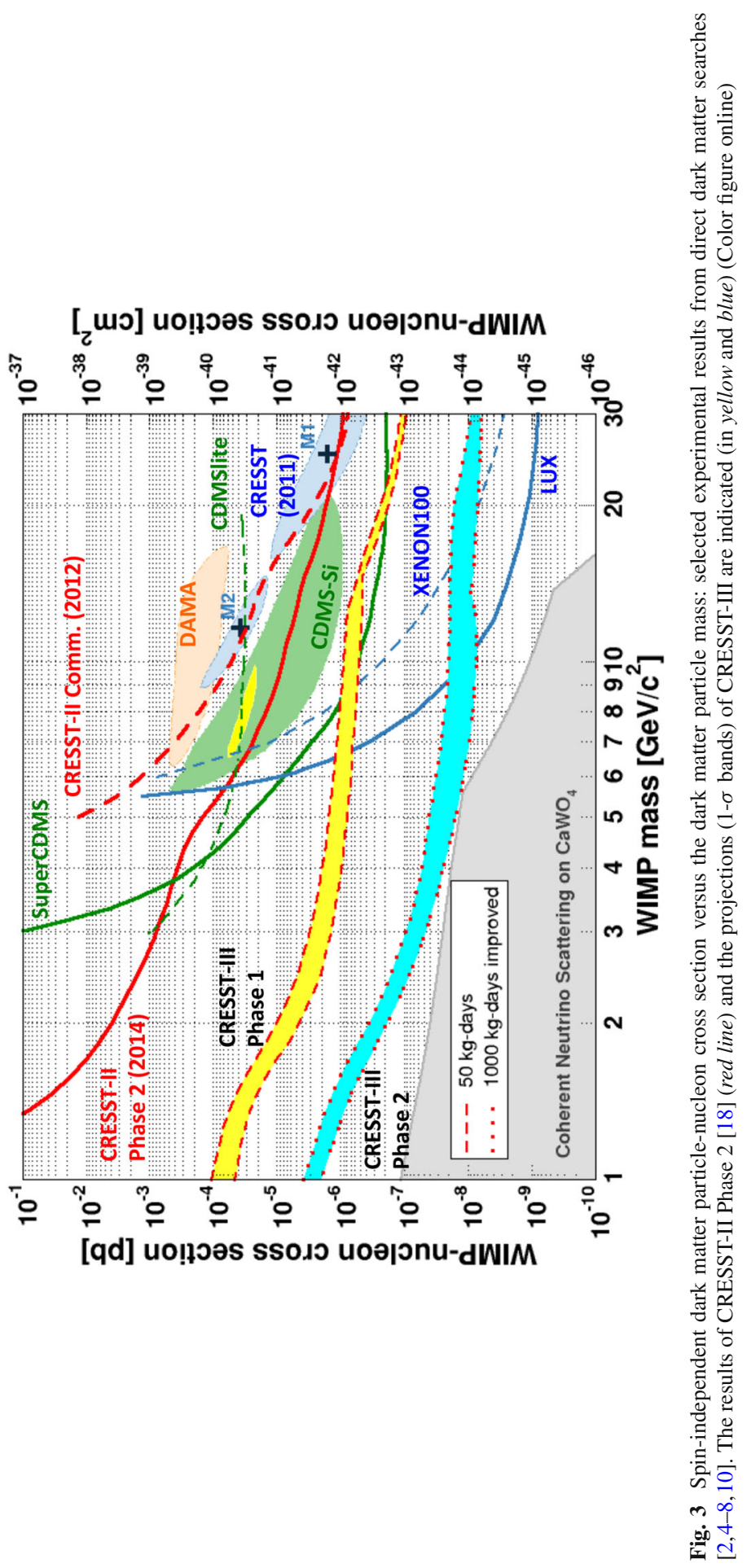


There are promising techniques to reduce, both, the intrinsic contamination of $\mathrm{CaWO}_{4}$ (e.g., by repeated re-crystallization at the growth facility at TUM) and the level of external radiation (e.g., by active and passive shielding around detectors, closepacking of detectors). Our goal is to lower the overall background by a factor of 100 for the second phase of CRESST-III. A sensitivity projection $(1 \sigma)$ for $1000 \mathrm{~kg}$-days of exposure of detectors with this improved performance is shown in Fig. 3 (blue band within dotted lines). This corresponds to 100 detectors of $24 \mathrm{~g}$ each measured for $\sim 2$ years which will require more readout channels.

\section{Conclusion}

The results of CRESST-II Phase 2 [18] demonstrate the potential of phonon-light detectors, in particular, for low-mass dark matter search. Energy thresholds of $\leq 100 \mathrm{eV}$ and significantly improved crystal-intrinsic background levels are feasible with the next generation of detectors. With a moderate target mass of $\mathcal{O}(3 \mathrm{~kg})$ and a measuring time of $\sim 2$ years, most of the accessible dark matter particle parameter space below $10 \mathrm{GeV} / \mathrm{c}^{2}$ can be probed. The preparations for the first phase of CRESST-III are ongoing and first data is expected for the beginning of 2016. On the long term, CRESST-III might reach sensitivities close to coherent neutrino-nucleus scattering cross sections with exposures of $\geq 50 \mathrm{~kg}$-years [21].

Acknowledgments Open access funding provided by Max Planck Society (or associated institution if applicable). This research was supported by the DFG cluster of excellence: Origin and Structure of the Universe, the DFG Transregio 27: Neutrinos and Beyond, the Helmholtz Alliance for Astroparticle Physics, the Maier-Leibnitz-Laboratorium (Garching), the Science \& Technology Facilities Council (UK), and by the BMBF: Project 05A11WOC EURECA-XENON. We are grateful to LNGS for the constant support of CRESST.

Open Access This article is distributed under the terms of the Creative Commons Attribution 4.0 International License (http://creativecommons.org/licenses/by/4.0/), which permits unrestricted use, distribution, and reproduction in any medium, provided you give appropriate credit to the original author(s) and the source, provide a link to the Creative Commons license, and indicate if changes were made.

\section{References}

1. CRESST Collaboration, G. Angloher et al., Results from $730 \mathrm{~kg}$ days of the CRESST-II Dark Matter search. Eur. Phys. J. C72, 41-22 (2012)

2. DAMA, LIBRA Collaboration, R. Bernabei et al., New results from DAMA/LIBRA. Eur. Phys. J. C67, 39-49 (2010). arXiv:1002.1028

3. CoGeNT Collaboration Collaboration, C. Aalseth et al., Cogent: a search for low-mass dark matter using p-type point contact germanium detectors. Phys. Rev. D88, 012002 (2013)

4. CDMS Collaboration Collaboration, R. Agnese et al., Silicon detector dark matter results from the final exposure of CDMS II. Phys. Rev. Lett. 111, 251301 (2013)

5. LUX Collaboration Collaboration, D. Akerib et al., First results from the LUX dark matter experiment at the Sanford Underground Research Facility. Phys. Rev. Lett. 112, 091303 (2014). arXiv:1310.8214

6. XENON100 Collaboration, E. Aprile et al., Dark matter results from 225 live days of XENON100 data. Phys. Rev. Lett. 109, 181301 (2012). arXiv:1207.5988

7. SuperCDMS Collaboration Collaboration, R. Agnese et al., Search for low-mass WIMPs with superCDMS. Phys. Rev. Lett. 112, 241302 (2014). arXiv: 1402.7137 
8. SuperCDMSSoudan Collaboration Collaboration, R. Agnese et al., CDMSlite: a search for low-mass WIMPs using voltage-assisted calorimetric ionization detection in the superCDMS experiment. Phys. Rev. Lett. 112, 041302 (2014). arXiv:1309.3259

9. EDELWEISS Collaboration, E. Armengaud et al., A search for low-mass WIMPs with EDELWEISS-II heat-and-ionization detectors, Phys. Rev. D86, 051701 (2012). arXiv:1207.1815

10. A. Brown, S. Henry, H. Kraus, and C. McCabe, Extending the CRESST-II commissioning run limits to lower masses. Phys. Rev. D85, 021301 (2012)

11. P. Cushman et al., Snowmass CF1 summary: WIMP dark matter direct detection. arXiv:1310.8327

12. J. Birks, The theory and practice of scintillation counting (Pergamon Press, New York, 1964)

13. R. Strauss et al., Energy-dependent light quenching in $\mathrm{CaWO}_{4}$ crystals at $\mathrm{mK}$ temperatures. Eur. Phys. J. C74, 7 (2014)

14. CRESST-II Collaboration, R. Strauss et al., A detector module with highly efficient surface-alpha event rejection operated in CRESST-II phase 2. Eur. Phys. J. C75, 352 (2015)

15. F. Reindl et al., Astroparticle, particle, space physics and detectors for physics applications, ch. 45, 290-296 (2014)

16. A. Erb, J.-C. Lanfranchi, Growth of high-purity scintillating $\mathrm{CaWO}_{4}$ single crystals for the lowtemperature direct dark matter search experiments CRESST-II and EURECA. CrystEngComm 15, 2301-2304 (2013)

17. CRESST-II Collaboration, R. Strauss et al., Beta/gamma and alpha backgrounds in CRESST-II Phase 2, JCAP 030 (2015)

18. CRESST-II Collaboration, G. Angloher et al., Results on low mass WIMPs using an upgraded CRESSTII detector. arXiv:1407.3146

19. F. Pröbst et al., Model for cryogenic particle detectors with superconducting phase transition thermometers. J. Low Temp. Phys. 100(1-2), 69-104 (1995)

20. M. Pyle, E. Figueroa-Feliciano, B. Sadoulet, Optimized designs for very low temperature massive calorimeters. ArXiv e-prints (2015) arXiv:1503.0120

21. A. Gütlein et al., Impact of coherent neutrino nucleus scattering on direct dark matter searches based on $\mathrm{CaWO}_{4}$ crystals, Astropart. Phys. 69, 44-49 (2015) 